\title{
Jones phase microscopy of transparent and anisotropic samples
}

\author{
Zhuo Wang, ${ }^{1}$ Larry J. Millet, ${ }^{2}$ Martha U. Gillette, ${ }^{2}$ and Gabriel Popescu ${ }^{1, *}$ \\ ${ }^{1}$ Quantitative Light Imaging Laboratory, Department of Electrical and Computer Engineering, Beckman Institute for \\ Advanced Science and Technology, University of Illinois at Urbana-Champaign, Urbana, Illinois 61801, USA \\ ${ }^{2}$ Department of Cell and Developmental Biology, University of Illinois at Urbana-Champaign, Urbana, \\ Illinois 61801, USA \\ *Corresponding author: gpopescu@uiuc.edu
}

Received February 26, 2008; revised April 28, 2008; accepted April 28, 2008; posted May 1, 2008 (Doc. ID 93177); published May 30, 2008

\begin{abstract}
We developed an interferometric microscopy technique, referred to as Jones phase microscopy, capable of extracting the spatially resolved Jones polarization matrix associated with transparent and anisotropic samples. This is a generalization of quantitative phase imaging, which is recovered from one diagonal element of the measured matrix. The principle of the technique is demonstrated with measurements of a liquid crystal spatial light modulator and the potential for live cell imaging with experiments on live neurons in culture. (C) 2008 Optical Society of America
\end{abstract}

OCIS codes: $180.0180,170.0170,120.0120$.

Polarization is the fundamental property of electromagnetic fields that describes the orientation of the oscillating electric field vector [1]. Recently, the theory of polarization has been generalized to include statistical behavior of optical fields [2,3]. Polarization-based techniques of investigation essentially probe the anisotropy in the induced charge displacement within a given sample and, thus, have the capability to sense molecular level organization. In the early 1940s, Jones developed a "field-based" $2 \times 2$ matrix formalism to describe the anisotropic response of a material in terms of its complex (i.e., phase and amplitude) behavior [4,5]. However, because polarization experiments have been largely limited to intensity measurements, the StokesMuller formalism, an intensity-based framework, has been commonly used instead (see [6] for a review).

Polarization-sensitive microscopy can reveal inner structures of cells without the need for exogenous contrast agents [12,13]. Quantifying the optical phase delays associated with live cells also gives access to intrinsic information about morphology and dynamics. Thus, quantitative phase imaging (QPI) has become an increasingly active field in recent years [14].

In this Letter we present a novel microscopy technique that is both quantitative in phase and polarization sensitive. Our method, referred to as Jones phase microscopy (JPM), extracts, for the first time to our knowledge, the full Jones matrix in each point within the field of view associated with a transparent sample. JPM uses a modified version of the Hilbert phase microscope (HPM) $[15,16]$. HPM is similar to the Leith-Upatnieks off axis analog holography $[17,18]$ that was later applied to digital holography $[19,20]$, in the sense that it uses spatial modulation to encode the phase information of the object. However, in HPM the measurement is performed in the image plane of a microscope rather than a Fresnel (i.e., out-of-focus) plane, which offers important advantages in the case of thin samples, as follows. First, the signal sampling, phase reconstruction, and unwrapping are more robustly performed in the image plane than in the Fresnel zone, where highfrequency interference patterns and phase discontinuities may occur. Second, in the image plane of a thin and transparent sample, such as live cells, the intensity is evenly distributed, which efficiently utilizes the limited dynamic range of the CCD. Finally, this approach does not require the numerical Fresnel propagation to the image plane, as the measured field is already in the image plane.

The experimental setup is shown in Fig. 1. He-Ne laser radiation is coupled into a 50/50 fiber coupler, and the two output beams are used as the arms of a Mach-Zehnder interferometer. On the object arm we use an inverted microscope (Meiji 5200) equipped with an $80 \times(\mathrm{NA}=0.9)$ objective to image the sample. This image is further relayed to the CCD (512 EMCCD, Princeton Instruments) via the L1-L2 lens system, with magnification of $2.5 \times$. The reference

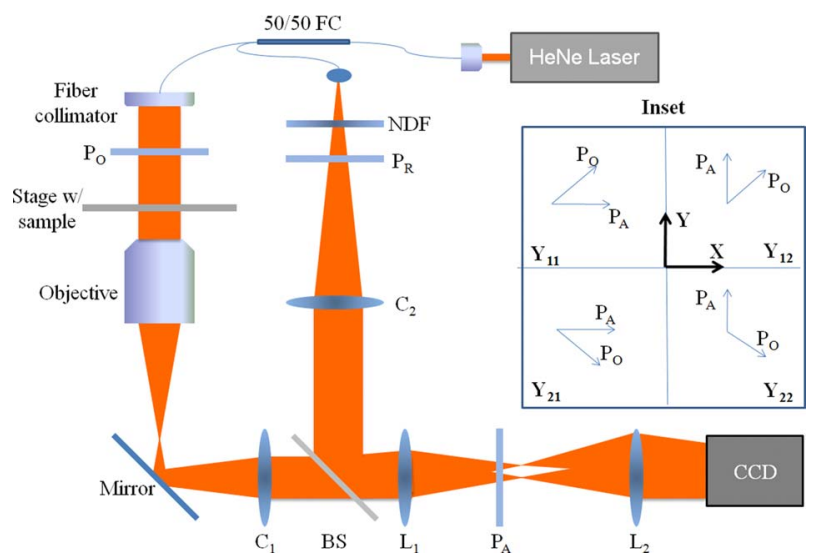

Fig. 1. (Color online) Jones phase microscope: $P_{O}, P_{R}$, and $P_{A}$, polarizers; $C_{1}, C_{2}$, collimating lenses; $\mathrm{L}_{1}, \mathrm{~L}_{2}$, Fourier lens pair; $\mathrm{BS}$, beam splitter; CCD, charge coupled device. Inset, polarization orientation for the four measurements. For $Y_{11}, P_{O}$ is at $45^{\circ}$ and $P_{A}$ is at $0^{\circ}$; for $Y_{12}, P_{O}$ is at $45^{\circ}$ and $P_{A}$ is at $90^{\circ}$; for $Y_{21}, P_{O}$ is at $-45^{\circ}$ and $P_{A}$ is at $0^{\circ}$; for $Y_{22}, P_{O}$ is at $-45^{\circ}$ and $P_{A}$ is at $90^{\circ} . P_{R}$ has the same orientation as $P_{O}$. 
beam is collimated, magnified by the same L1-L2 system, and makes a small angle with the object beam, such that interference fringes are generated at the CCD $(512 \times 512$ pixels per frame $)$. The intensity recorded has the following form:

$$
I(x)=I_{R}+I_{S}(x)+2\left[I_{R} I_{S}(x)\right]^{1 / 2} \cos [q x+\phi(x)],
$$

where $I_{R}$ and $I_{S}$ are the reference and the sample irradiance distributions, $q$ is the spatial frequency of the fringes, and $\phi$ is the spatially varying phase associated with the object. These fringes are typically sampled by $5-6$ pixels, which fulfills the sampling requirement for preserving the optical resolution of the microscope [21]. The HPM uses the basic principle of reconstructing a complex analytic signal from its associated real part. The complex analytic signal formalism was first introduced in optics by Gabor [22] In the spatial domain this type of reconstruction became practical with the advancement of computer algorithms for fast Fourier transformation, as demonstrated by Takeda et al. [23] and later applied to microscopy by Cuche et al. [24]. Thus, the quantitative phase image associated with the sample is reconstructed using a 2D spatial Hilbert transform, as described in $[15,16]$.

To control the polarization of the beams on each arm we used two polarizers, $P_{O}$ and $P_{R}$, which transform the elliptical polarizations of the fields from the optical fiber into linear polarization along controllable directions. In the laboratory system of reference, the Jones matrix of an arbitrary sample is defined as

$$
\underline{J} \equiv\left[\begin{array}{ll}
J_{x x} & J_{x y} \\
J_{y x} & J_{y y}
\end{array}\right],
$$

where the matrix elements are complex numbers. To interrogate the sample we prepare two incident electric vectors, oriented at $+45^{\circ}$ and $-45^{\circ}$ with respect to the reference axis:

$$
\begin{gathered}
E_{+45}=C_{1}\left[\begin{array}{l}
1 \\
1
\end{array}\right], \\
E_{-45}=C_{2}\left[\begin{array}{l}
1 \\
-1
\end{array}\right],
\end{gathered}
$$

with $C_{1,2}$ real constants. We perform four sets of measurements, each containing phase and amplitude images $\left(Y_{i j}\right.$ complex, $\left.i, j=1,2\right)$, corresponding to the analyzer $P_{A}$ oriented parallel and perpendicular with respect to the two directions:

$$
\begin{aligned}
& {\left[\begin{array}{l}
Y_{11} \\
Y_{12}
\end{array}\right]=C_{1}\left[\begin{array}{l}
J_{x x}+J_{x y} \\
J_{y x}+J_{y y}
\end{array}\right],} \\
& {\left[\begin{array}{l}
Y_{21} \\
Y_{22}
\end{array}\right]=C_{2}\left[\begin{array}{l}
J_{x x}-J_{x y} \\
J_{y x}-J_{y y}
\end{array}\right] .}
\end{aligned}
$$

We can rewrite Eqs. (4a) and (4b) in a compact form by stacking them into a single $4 \times 4$ matrix equation:

$$
\left[\begin{array}{l}
Y_{11} \\
Y_{21} \\
Y_{12} \\
Y_{22}
\end{array}\right]=\left[\begin{array}{llll}
C_{1} & C_{1} & 0 & 0 \\
C_{2} & -C_{2} & 0 & 0 \\
0 & 0 & C_{1} & C_{1} \\
0 & 0 & C_{2} & -C_{2}
\end{array}\right]\left[\begin{array}{l}
J_{x x} \\
J_{x y} \\
J_{y x} \\
J_{y y}
\end{array}\right] .
$$

The inset of Fig. 1 describes the four polarization combinations. The four complex elements of the Jones matrix are obtained by inverting the $4 \times 4$ matrix $(C)$ in Eq. (5). The constants $C_{1}$ and $C_{2}$ are retrieved by performing the measurement with no sample, i.e., with $J$ as the identity $2 \times 2$ matrix. This procedure is robust and works for an arbitrary transparent and anisotropic sample, for which the condition $\operatorname{det}(C) \neq 0$ always holds.

We demonstrated the principle of operation of JPM with measurements of the Jones matrix associated with a controllable spatial light modulator (SLM). The transmission SLM is made of a twisted nematic liquid crystal that is controlled via the red channel of red-green-blue (RGB) video input. A vertical polarizer is placed in front of the SLM and aligned with its principal axis, such that the expected Jones matrix contains nonzero terms in the right column only. The result of this measurement is shown in Fig. 2. It can be seen that the amplitude maps in the left column are close to the expected zero level, to within the noise level. In addition, the phase maps on this column are very noisy, as expected, because they are associated with fields of very low amplitudes where phase is not well defined. In the right column of the SLM Jones matrix, both amplitudes and the phase maps are reconstructed with high signal to noise ratio and show the expected pattern inputted via the RGB signal. To find the phase relationship between $J_{x y}$ and $J_{y y}$, we performed an additional intensity measurement with the analyzer placed at $45^{\circ}$ such that the two terms are coupled.

We proved the ability of JPM to extract Jones matrices associated with biological samples by imaging live neurons in culture. Primary hippocampal rat neurons were established through a previously published procedure [25]. Figure 3 shows the Jones matrix maps of a single neuron. As can be seen, the amplitude of the diagonal terms shows very little contrast, attesting that the cell is transparent. By contrast, the diagonal phase maps reveal the structure of the neuron with soma (cell body) and several processes (i.e., axon or dendrites) clearly visible. The difference between these two phase images is very small, proving that the birefringence is negligible in this case. The off diagonal elements show zero amplitudes and structureless phase distribution, which indicate a lack of measurable polarization effects.

In summary, JPM is a new direct technique to extract the Jones matrix of a transparent and anisotropic sample in a spatially resolved manner. The experiment described here is carried out in transmission geometry. However, the procedure is equally applicable for a reflective type illumination. The authors are currently working to improve the sensitivity of the technique to polarization changes 


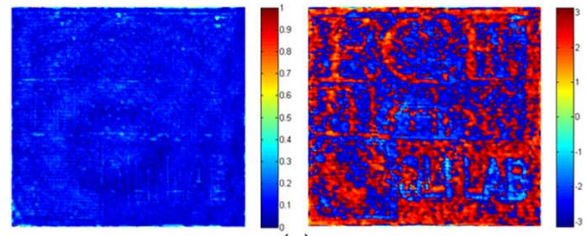

(a)

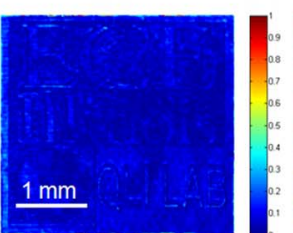

(c)

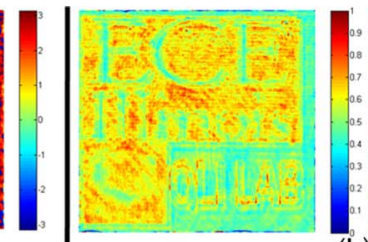

(b)

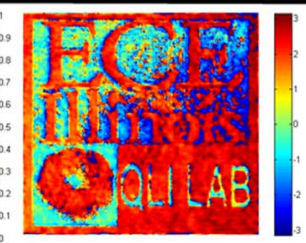

(d)
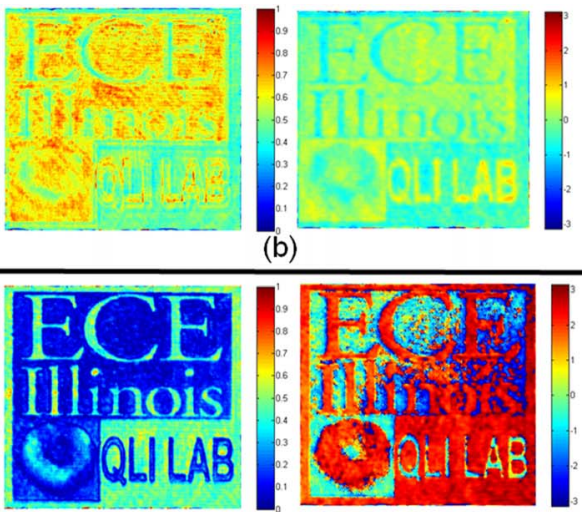

Fig. 2. (Color online) Amplitude and phase maps of the Jones matrix for the SLM-polarizer combination. For each subplot, left is the amplitude map (bar in arbitrary units) and right is the phase map (bar in radians). The scale bar is the same in all images. (a) $J_{x x} ;$ (b) $J_{x y} ;$ (c) $J_{y x} ;$ (d) $J_{y y}$.
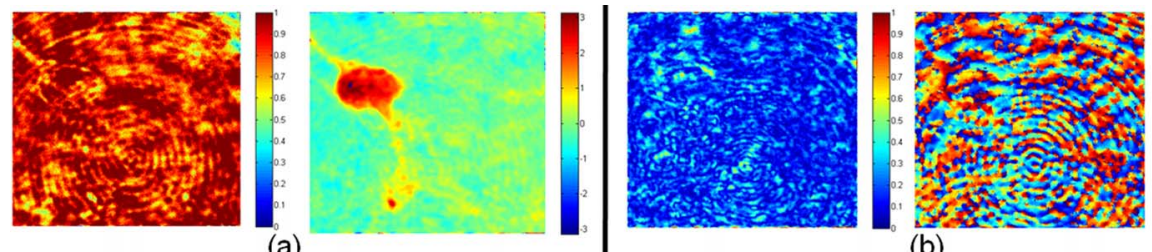

(b)

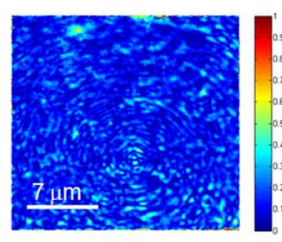

a)

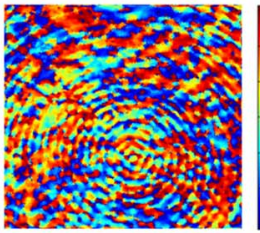

(c)

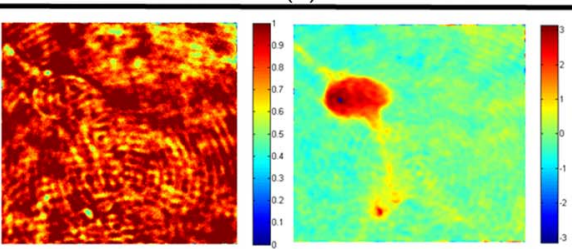

(d)

Fig. 3. (Color online) JPM of an individual hippocampal neuron (live) in cell culture. For each subplot, the left side is the amplitude map (bar in arbitrary units) and the right side is the phase map (bar in radians). (a) $J_{x x}$; (b) $J_{x y}$; (c) $J_{y x}$; (d) $J_{y y}$.

and to make JPM amenable to dynamic studies of transparent and anisotropic systems.

Z. Wang and G. Popescu thank Dan Marks for stimulating discussions.

\section{References}

1. A. Yariv and P. Yeh, Optical Waves in Crystals: Propagation and Control of Laser Radiation (WileyInterscience, 2002).

2. E. Wolf, Phys. Lett. A 312, 263 (2003).

3. J. Tervo, T. Setala, and A. T. Friberg, Opt. Express 11, 1137 (2003).

4. R. C. Jones, J. Opt. Soc. Am. 31, 6 (1941).

5. R. C. Jones, J. Opt. Soc. Am. 37, 3 (1947).

6. M. Mujat, "Polarimetric characterization of random electromagnetic beams and applications," Ph.D dissertation (University of Central Florida, 2004).

7. J. F. de Boer and T. E. Milner, J. Biomed. Opt. 7, 359 (2002).

8. V. Backman, R. Gurjar, K. Badizadegan, L. Itzkan, R. R. Dasari, L. T. Perelman, and M. S. Feld, IEEE J. Sel. Top. Quantum Electron. 5, 1019 (1999).

9. J. M. Schmitt, A. H. Gandjbakhche, and R. F. Bonner, Appl. Opt. 31, 6535 (1992).

10. B. D. Cameron, M. J. Rakovic, M. Mehrubeoglu, G. W. Kattawar, S. Rastegar, L. V. Wang, and G. L. Cote, Opt. Lett. 23, 485 (1998).

11. H. Ding, J. Q. Lu, R. S. Brock, T. J. McConnell, J. F.
Ojeda, K. M. Jacobs, and X. H. Hu, J. Biomed. Opt. 12, 034032 (2007).

12. R. Oldenbourg, Nature 381, 811 (1996).

13. M. Pluta Advanced Light Microscopy: Measuring Techniques (North Holland, 1993).

14. G. Popescu, "Quantitative phase imaging and applications: a review," (2007) http://light.ece.uiuc.edu/ QPIreview.htm.

15. T. Ikeda, G. Popescu, R. R. Dasari, and M. S. Feld, Opt. Lett. 30, 1165 (2005).

16. G. Popescu, T. Ikeda, C. A. Best, K. Badizadegan, R. R. Dasari, and M. S. Feld, J. Biomed. Opt. 10, 060503 (2005)

17. E. N. Leith and J. Upatnieks, J. Opt. Soc. Am. 51, 1123 (1962).

18. E. N. Leith and J. Upatnieks, J. Opt. Soc. Am. 53, 1377 (1963).

19. B. R. Brown and A. W. Lohmann, Appl. Opt. 5, 967 (1966).

20. J. W. Goodman and R. W. Lawrence, Appl. Phys. Lett. 11, 77 (1967).

21. J. W. Goodman Introduction to Fourier Optics (McGraw-Hill, 1996).

22. D. Gabor, J. Inst. Electr. Eng. 93, 329 (1946).

23. M. Takeda, H. Ina, and S. Kobayashi, J. Opt. Soc. Am. 72, 156 (1982).

24. E. Cuche, P. Marquet, and C. Depeursinge, Appl. Opt. 39, 4070 (2000).

25. L. J. Millet, M. E. Stewart, J. V. Sweedler, R. G. Nuzzo, and M. U. Gillette, Lab Chip 7, 987 (2007). 